\title{
Energy Consumption Models For MISO-UWB and TR-MISO-UWB Systems
}

\author{
Adil El Abboubi, Fouzia Elbahhar, Marc Heddebaut, and Yassin Elhillali
}

\begin{abstract}
-this paper, an energy consumption model is developed and exploited to evaluate the electrical energy consumption of ultra-wideband impulse radio (UWB-IR) systems. We develop the energy consumption models and our comparative study, on the one hand, for a system based single-input singleoutput (SISO) configuration and a multiple-input single-output (MISO) and, on the other hand, for a time reversal TR-MISO configuration and for MISO alone configuration. We consider an indoor propagation environment based on the 802.15.4a channel model. The results show very different behaviors depending on the propagation conditions, the number of antennas used, or on the number of transmitted symbols. Using such a model, a radiofrequency designer can obtain significant inputs to optimally select an adequate configuration to design an adaptive energyaware UWB-IR system.
\end{abstract}

Keywords-energy efficiency, time reversal, MISO systems, UWB

\section{INTRODUCTION}

$\mathbf{U}$ LTRA-WIDEBAND impulse radio (UWB-IR) is a wireless communication technology that uses short pulses whose durations are typically less than $1 \mathrm{~ns}$. They transmit radiofrequency signals over a very large bandwidth, greater than or equal to $500 \mathrm{MHz}$. Due to these characteristics, UWB permits high data rates transmission for communication systems and also high performance for radar and localization applications. However, existing regulations strictly limit the level of transmission power to, for example, $-41.3 \mathrm{dBm} / \mathrm{Hz}$ in some parts of the world and, therefore, restrict UWB operation to limited ranges. In order to increase the channel capacity, the communication range, the robustness or the quality of the transmission, some authors integrate MIMO configurations to UWB systems [1]. Due to its intrinsic characteristics, the UWB technique is mainly used in indoor environments characterized by multipath propagation conditions. In such propagation conditions, MIMO architecture often offers some of the above mentioned benefits. However the energy consumption of MIMO systems is potentially higher than the energy required by corresponding SISO systems since they necessitate multiple $\mathrm{RF}$ chains and require additional signal processing [2]. In this paper we develop the energy consumption models in order to evaluate the difference. The authors in [3] reviewed the methods of measuring and modelling MIMO-UWB systems and they described the different results obtained during the

A. Elabboubi was with the the french institute of science and technology for transport, development and networks, 20 rue Elise Reclus, 59666 Villeneuve dAscq cedex, France (e-mail: aelabboubi@gmail.com)

F. Elbahhar and M. Heddebaut are with the the french institute of science and technology for transport, development and networks (e-mail: fouzia.boukour@ifsttar.fr,marc.heddebaut@ifsttar.fr)

Y. Elhillali is with the university of Valenciennes (email yassin.elhillali@univ-valenciennes.fr) measurement campaigns related to each considered model. As the two propagation models studied in this paper are the Saleh-Valenzuela $(\mathrm{S}-\mathrm{V})$ model with directional information or a geometry-based stochastic model, they concluded that the correlation and the channel capacity of the UWB-MIMO systems vary according to the variation of the frequency range. The paper [4] describes the measurements of an emulated indoor channel for $2 \times 2,2 \times 3,2 \times 4$ UWB-MIMO systems. The capacities of different MIMO-UWB systems were calculated and showed that the MIMO-UWB provides a better capacity as compared to the SISO-UWB. Malik and Edwards [5] investigated the robustness and rate enhancement due to the use of spatial and polar array in MIMO-UWB systems. They conducted their experiments in an indoor channel and they concluded that the polar arrays suffer less from correlation than the spatial ones, and despite the compactness of the polar systems their achievable capacity and SNR gain are slightly less than the systems using spatial arrays. In [6] the authors study the impact of the correlation on a UWB-MIMO system using a TR technique. They showed that the system combining MIMO-UWB and TR outperforms the conventional UWBMIMO system under severe correlation conditions in indoor environment for both Line Of Sight (LOS) and Non Line Of Sight (NLOS). In the reference [7], the authors used TR associated to a MIMO-UWB system to tackle the range limitation due to the power limitation imposed by the regulatory bodies. They showed that the TR-MIMO-UWB can achieve a significant power gain depending on the number of antennas used, while keeping the complexity of the receiver low. In [8], we evaluated the energy consumption of UWB systems and we showed that the combination of a UWB system and a TR technique may reduce the overall energy consumed by the system for all the modulation techniques considered. As a further contribution to the UWB-MIMO research, the study of the total energy consumption of MIMO-UWB systems merits consideration since they can be integrated in many energyaware devices. In this paper, we concentrate on an energetic model for MISO-UWB systems. The model includes the power transmission and the power consumed by the circuitries of the transceiver. We shall use it to compare the energy consumption of MISO-UWB systems and of the corresponding SISOUWB systems. Moreover, the energy consumption of MISOUWB systems and systems that combine MISO-UWB and TR technique are also compared in order to analyse the impact or the contribution that TR can have or add to the total energy consumption of the system. This evaluation takes into account the multipath propagation effects of an indoor environment based on the 802.15.4a channel model [9]. 
Considering this approach, the rest of the paper is organized as follows. We discuss UWB based MISO system in section II. In section III, we present the energetic model. Section IV provides the channel description. In section $\mathrm{V}$, we express the energy efficiency of the MISO-UWB system. We analyse the energy consumption of the TR-MISO-UWB in section VI. We present the results of comparison between MISO and SISO systems in section VII. In section VIII, we illustrate the comparison of the MISO and TR-MISO systems. Finally, a conclusion and perspectives are drawn.

\section{MISO-UWB MODEL}

A MISO system that uses an array of $\mathrm{N}$ transmitting antennas and one antenna for reception is depicted in Fig. 1.

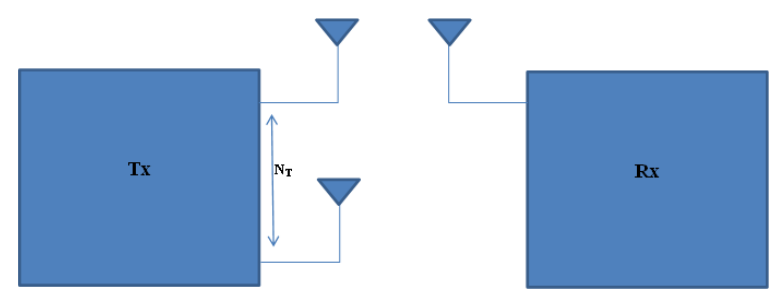

Fig. 1. MISO system simplified representation

Among the different possible MIMO architectures, the MISO architecture is known for its increased performances including data rate due to the effective use of spatial diversity [1]. Furthermore, MISO systems enhance the focalization capacity of TR systems [10] that will also be considered in our comparative energetic evaluation.

\section{ENERGETIC MODEL}

To study the energy consumption of a wireless communication system, we exhaustively consider the electronic components used in the telecommunication chain. Sending a sequence of $\mathrm{N}$ bits demands a time duration noted $\mathrm{T}$. We assume the transceiver works according to three different operating modes:

- Active mode: in this mode, the information is transmitted. The time needed by the transceiver to send the sequence of bits is noted $T_{a c}$. In this case, all the components are working and consuming power.

- Sleep mode: when there is no information to convey, the system enters a sleep mode. The time spent by the transceiver in standby is noted $T_{s l}$. In this case, a restricted number of necessary components are active and consuming power.

- Transient mode: this corresponds to the transition mode between the sleep mode and the active mode. The time spent by the transceiver to shift from standby to full functioning is noted $T_{t r}$.

Thus, $T$ can be written according to (1):

$$
T=T_{a c}+T_{s l}+T_{t r}
$$

In [11] the authors did not take into account the time to shift from active to sleep mode because it is usually very fast compared to the shift from sleep to active mode. However, they still consider the amount of time spent by the system to shift from sleep to active mode due to the use of a synthesizer, which is energy consuming when the transition takes place. In our case, for the UWB-IR technique, we do not use a synthesizer. So, we do not take into account this transient time. Therefore, the energy needed to transmit $\mathrm{N}$ bits is given by (2):

Thus, $\mathrm{T}$ can be written according to (1):

$$
E=P_{a c} T_{a c}+P_{s l} T_{s l}
$$

$P_{a c}$ and $P_{s l}$ represent the power consumption values during the active mode and the sleep mode respectively. The power consumption of the active mode includes the transmission power $\mathrm{Pt}$ and the electronic circuitry power consumption $P_{c}$. $P_{c}$ combines the receiver power consumption noted $P_{c r}$ and the transmitter power consumption noted $P_{c t}$. In a further section, we shall detail the composition of these powers because the associated hardware components may vary according to the selected transmission technique. In the transmission part, we also use a power amplifier noted PA. Its power consumption is linked to the transmission power by:

$$
P_{a m p}=a P_{t}
$$

Where $a=\xi / \eta-1$, with $\xi$ the average of peak to ratio and $\eta$ the drain efficiency of the PA. These variables depend on the operating class of the amplifier and of the selected modulation scheme. As compared to the power consumption in the active mode, the power consumption in the sleep mode is very low. Therefore, in this study, we also assume that $P_{s} l=0$. However, it could be considered for specific applications necessitating long periods in sleep mode [10]. Finally, the amount of energy needed to transmit one bit of information is given in (4):

$$
E_{T}=\frac{\left[(1+a) P_{t}+\left(P_{c}-P_{a m p}\right)\right] T a c}{N}
$$

$\mathrm{N}$ represents the total number of bits to be transferred.

\section{CHANNEL DESCRIPTION}

For the MISO system operating in an indoor environment, we adapt the channel model introduced in [1]. This channel model describes the MISO channel as a sum of independent channels where the time delay between the different paths for all the channels is the same. In the model we use, in order to be more representative of a real environment, we change this assumption slightly and we choose a different time delay for each channel. This model can be expressed by (5):

$$
y(t)=\sum_{l=1}^{L} \sum_{i=1}^{N_{T}} \alpha_{l, i} x_{i}\left(t-(l-1) \tau_{i}\right)+n(t)
$$

The vectors $y(t)$ and $n(t)$ represent the received signal and the white Gaussian noise respectively. $x_{i}(t)$ is the symbol transmitted by the antenna number $i . \alpha_{l, i}$ is the multipath component (MPC) of the path 1 linked to the antenna $i . \tau_{i}$ is the propagation delay of the first path of the antenna $i$ 
with $\tau_{i}=\tau_{1}+(i-1) d_{A} / c$, where $\tau_{1}$ is the propagation delay of the first path of the antenna 1 and $d_{A}$ is the distance between two neighboring antennas and $\mathrm{c}$ is the speed of light. We assume that the propagation delay $i$, noted $\tau_{i}$, of channel 1 , corresponding to antenna $i$, is longer than the pulse duration $T_{p}$. As a consequence, the intra-symbol interferences (ISI) are cancelled [12]. We consider a Nakagami-m distribution for the MPCs as described by the 801.15.4a task group [9]. For a limited number of paths $L_{p}<L$ where the $L_{p}$ paths have the same average signal to noise ratio (SNR) $\bar{\gamma}_{i}$, the instantaneous average power density function of the SNR for the antenna $i$ can be written as in (6) [13]:

$$
f_{\gamma_{i}}\left(\gamma_{i}\right)=\frac{\gamma_{i}^{m L_{p}-1}}{\Gamma\left(m L_{p}\right)\left(\frac{\bar{\gamma}_{i}}{m}\right)^{m L_{p}}} \exp \left(\frac{-m \gamma_{i}}{\bar{\gamma}_{i}}\right)
$$

Where $m$ is the shape parameter of the Nakagami-m distribution and $\Gamma(m)=\int_{0}^{+\infty} x^{m-1} e^{-x} d x$ is the gamma function with $\operatorname{Re}(\Gamma(m))>0$. We can write the instantaneous SNR for the antenna $\mathrm{i}$ as $\gamma_{i}=\sum_{l=0}^{L-1} \gamma l, i$ with $\gamma_{i}=\frac{\alpha_{l, i}^{2} P_{t_{i}}}{G_{d} N_{0} B}$ and:

$$
\bar{\gamma}_{i}=\frac{\Omega_{i} P_{t_{i}}}{G_{d} N_{0} B}
$$

Where $\Omega_{l, i}=E\left[\alpha_{l, i}^{2}\right], \Omega_{i}$ is the average of $\Omega_{l, i}$. We can express the power delay profile using (8) [14]:

$$
\Omega_{l, i}=\exp \left(\frac{-(l-1) \Delta \tau_{i}}{\bar{\sigma}}\right)
$$

With $\Delta \tau_{i}$ is the delay between two MPCs and $\bar{\sigma}$ is the RMS delay spread linked to the environment of the transmission.

\section{ENERGY EFFICIENCY OF THE MISO-UWB SYSTEM}

The instantaneous symbol error rate is given by (9) [15]:

$$
P_{M, i}\left(\gamma_{i}\right)=g_{1} Q\left(\sqrt{g_{2} \gamma_{i}}\right)
$$

The values of the parameters $g_{1}$ and $g_{2}$ determine the type of modulation. The total symbol error rate of the antenna $i$ can be written as (9):

$$
P_{M, i}=\int_{0}^{+\infty} P_{M, i}\left(\gamma_{i}\right) f_{\gamma_{i}}\left(\gamma_{i}\right) d \gamma_{i}
$$

We can write:

$$
P_{M, i}\left(\gamma_{i}\right) \leq \frac{g_{1}}{2} \exp \left(\frac{g_{2} \gamma_{i}}{2 m}\right)
$$

Thus, by replacing (11) and (6) in (10), we obtain the following upper bound of the total symbol error rate of antenna $i$ :

$$
P_{M, i}\left(\gamma_{i}\right) \leq \frac{g_{1}}{2}\left(1+\frac{g_{2} \bar{\gamma}_{i}}{2}\right)^{-m L_{p}}
$$

Finally, by using expressions (12) and (7) we get the approximation of the total symbol error rate of antenna $i$ given by (13):

$$
P_{M, i}\left(\gamma_{i}\right) \approx \frac{g_{1}}{2}\left(1+g_{2} c_{i} P_{t_{i}}\right)^{-m L_{p}}
$$

Where $c_{i}=\frac{\Omega_{i}}{G_{d} N_{0} B}$

For MISO-UWB systems between $3.6 \mathrm{GHz}$ and $5.1 \mathrm{GHz}$, the correlation between the transmission antennas is significantly mitigated when the distance between the neighboring antennas $d_{A} \geq 10 \mathrm{~cm}$ as mentioned in[16]. Therefore, we choose $d_{A}=10 \mathrm{~cm}$ in our application and assume that the correlation between the antennas is nil. In this case, the total symbol error rate of a MISO-UWB system is: $P_{M}=\sum_{i=1}^{N_{T}} P_{M, i}$.

We also assume that the power control is perfect, and then we can write that $P_{t}=P_{t_{i}}$. Finally, the total symbol error rate can be expressed by (14):

$$
P_{M}=\sum_{i=1}^{N_{T}} \frac{g_{1}}{2}\left(1+g_{2} c_{i} P_{t_{i}}\right)^{-m L_{p}}
$$

In order to compute the transmission power $\mathrm{Pt}$ for a given total symbol error rate, we must solve numerically (15):

$$
\sum_{i=1}^{N_{T}} \frac{g_{1}}{2}\left(1+g_{2} c_{i} P_{t_{i}}\right)^{-m L_{p}}-P_{M}=0 ;
$$

This leads us to the final equation (15) that will be used to compute the total energy consumption of a MISO-UWB system using a rake receiver with LR fingers:

$$
\begin{aligned}
E_{M I S O-U W B} & =\left(N_{T}(1+a)+N_{T}\left(P_{c t}-P_{a m p}\right)\right) T_{a c} \\
& +P_{c r} T a c
\end{aligned}
$$

With $\mathrm{a}$ is the coefficient linked to the power amplifier and $T_{a c}$ is the active time. Such a system is represented in Fig. 2.

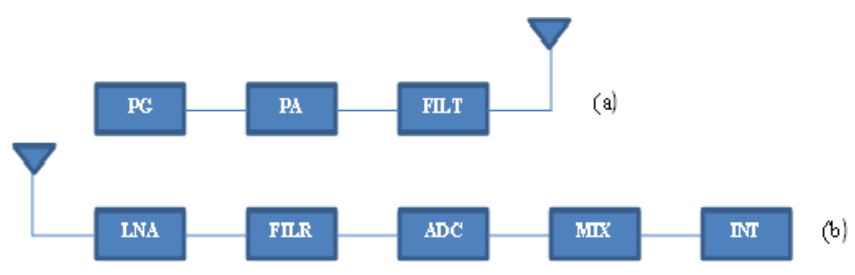

Fig. 2. (a) transmitter architecture, (b) receiver architecture

To compute the transmission power of this system, we have to replace $L_{p}$ in (14) by $L_{R}$ (the rake receiver fingers) and replace the powers of the transceiver circuitries by the following equations:

$$
P_{c t}=P_{p g}+P_{a m p}+P_{f i l t}
$$

$$
P_{c r}=P_{L N A}+L_{R}\left(P_{m i x}+P_{i n t}\right)+P_{f i l r}+P_{A D C}
$$

\section{ENERGY EFFICIENCY OF TR-MISO-UWB}

A conventional time reversal (TR) communication technique consists in pre-filtering the signal by the reversed complex conjugate of the channel impulse response (CIR). Then, the output signal after the filtering is convolved with the channel 
IR as in Fig.3. So the TR technique is expressed by the following:

$$
y(t)=s(t) \otimes h(t)^{e q}+n(t)
$$

where $\otimes, *$ and $h(t)^{e q}=h(-t)^{*} \otimes h(t)$ respectively denote convolution, complex conjugate and the autocorrelation of the CIR.

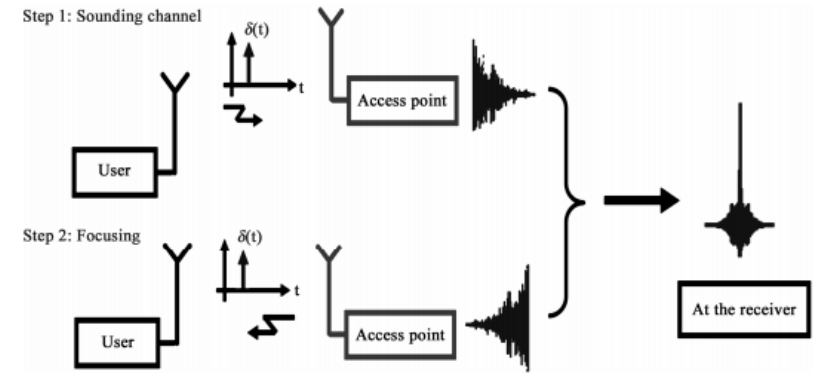

Fig. 3. Time reversal principle

In [8] we showed that combining the time reversal (TR) technique and the UWB decreases the total energy consumption of the system. Hence, in this section, we evaluate the total energy consumption of the TR-MISO versus the total energy consumption of the MISO system. In order to compute the transmission energy of the TR-MISO, we replace $L_{P}$ by $L_{T} R$, the number of paths considered to implement the TR technique, in (15), and we resolve the equation numerically. For calculating the power consumption of the TR-MISO transceiver, we use the following equations:

$$
\begin{gathered}
P_{c t}=P_{p g}+P_{a m p}+P_{f i l t}+P_{m i x}+P_{i n t} \\
P_{c r}=P_{L N A}+L_{R}\left(P_{m i x}+P_{i n t}\right)+P_{f i l r}+P_{A D C}
\end{gathered}
$$

The TR pre-filtering transmitting block is composed of a mixer and an integrator. Concerning the receiver, the TRMISO circuitry does not need a rake receiver to intercept the different paths. This comes from the intrinsic focusing properties of the TR technique.

\section{MISO VS SISO}

In this section, we compare the energy consumption of the MISO 2x1 system versus the energy consumption of a SISO system. For both MISO and SISO systems, we use an Mary Pulse Amplitude Modulation (MPAM) to modulate the signal (the energy efficiency of UWB systems using different modulation technique was evaluated in [17]). The modulation and delay parameters associated to equation (15) are given in table I.

TABLE I

MOdulation AND DELAY PARAMETERS

\begin{tabular}{lll}
\hline$g_{1}=\frac{2 M}{M-1}$ & $g_{2}=\frac{6}{M^{2}-1}$ & $\tau_{1}=0.7$ \\
\hline
\end{tabular}

To compute the total energy consumption of the SISO system, we use the following equation:

$$
\begin{aligned}
E_{S I S O} & =(1+a) m\left(\frac{M^{2}-1}{3}\right)\left(\left(\frac{M P_{M}}{M-1}\right)^{\frac{-1}{m L_{R}}}-1\right) \frac{N_{0} G_{d} N}{\Omega \log _{2}(M)} \\
& +\frac{\left(P_{c}-P_{a} m p\right) T_{a} c}{N}
\end{aligned}
$$

As stated before, this study is performed in an indoor environment and we use relevant IEEE 802.15.4a standard parameters. We compute the energy efficiency of the systems in both line of sight (LOS) case and non-line of sight (NLOS) conditions. The powers associated to the circuitry blocks and some other common parameters to the environment are indicated in table II.

TABLE II

SYSTEM PARAMETERS

\begin{tabular}{ccc}
\hline \hline$f_{c}=5 \mathrm{GHz}$ & $f=3.85 \mathrm{Ghz}$ & $B=1.5 \mathrm{GHz}$ \\
$N=10^{6}$ & $P_{p g}=25.2 \mathrm{~mW}$ & $P_{L N A}=7.68 \mathrm{~mW}$ \\
$P_{\text {mix }}=15 \mathrm{~mW}$ & $P_{\text {int }}=2.5 \mathrm{~mW}$ & $P_{L N A}=7.6 \mathrm{~mW}$ \\
$P_{\text {filt }}=2.2 \mathrm{~mW}$ & $P_{\text {pre-filt }}=2.5 \mathrm{~mW}$ & $P_{\text {filr }}=2.5 \mathrm{~mW}$ \\
$P_{M}=10^{-3}$ & $a=0.78$ & $N_{0}=-170 \mathrm{dBm} / \mathrm{Hz}$ \\
$\Delta \tau=0.7$ & $\beta=500$ & \\
\hline
\end{tabular}

These values correspond to realistic current technology parameters. We assume that the number of taps $L=100$ is the same for both LOS and NLOS cases.

The considered channel parameters and path-loss are presented in table III.

TABLE III

INDOOR ENVIRONMENT PARAMETERS

\begin{tabular}{c|c}
\hline \hline LOS & NLOS \\
$n=1.79$ & $n=4.89$ \\
$k=1.24$ & $k=1.85$ \\
$P L_{0}=43.9 d B$ & $P L_{0}=48.7 d B$ \\
$m=0.67 d B$ & $m=0.69 d B$ \\
$\Omega=0.93$ & $\Omega=0.94$ \\
$\overline{\sigma_{\tau}}=14$ & $\overline{\sigma_{\tau}}=18$ \\
\hline \hline
\end{tabular}

In Fig. 4 and Fig. 5, we present the total energy consumption of the MISO system and the SISO system versus the distance between the transmitter and the receiver, where $d=[5,20 \mathrm{~m}]$ for respectively both LOS and NLOS cases. We use $P_{M}=$ $10^{-3}, M=2$ and $L_{R}=4$. In LOS conditions, the SISO system consumes less than the MISO system and the energy consumption remains constant over the whole communication range considered. In this situation, the requested electrical energy is essentially consumed by the circuitries and appears constant over the communication range selected.

In NLOS conditions, we also obtain that the SISO system is more energy efficient than the MISO system. However, in these propagation conditions, the total energy consumption varies with respect to the communication range. Therefore, in this case, we obtain a consequent increase of consumed energy as 


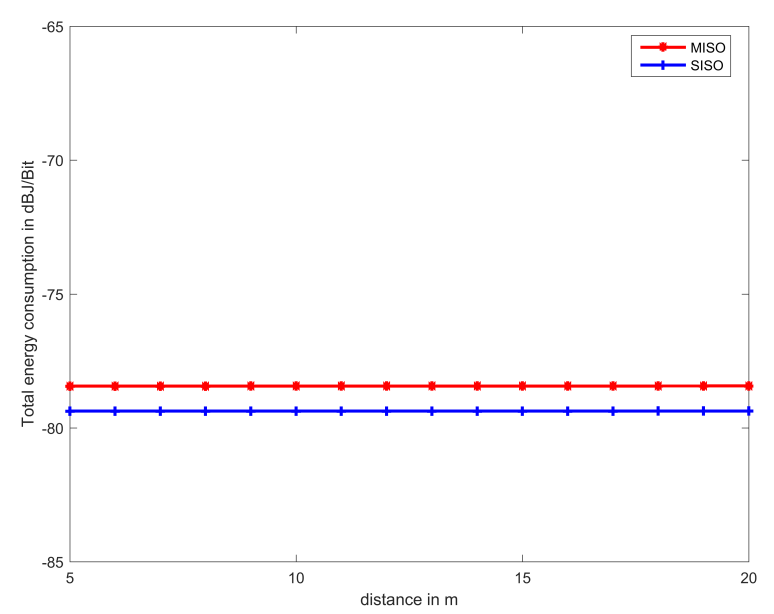

Fig. 4. Total energy consumption for MISO $2 \times 1$ and SISO with $d=[5,20 m], P_{M}=10^{-3}$ and $M=2$ for the LOS case

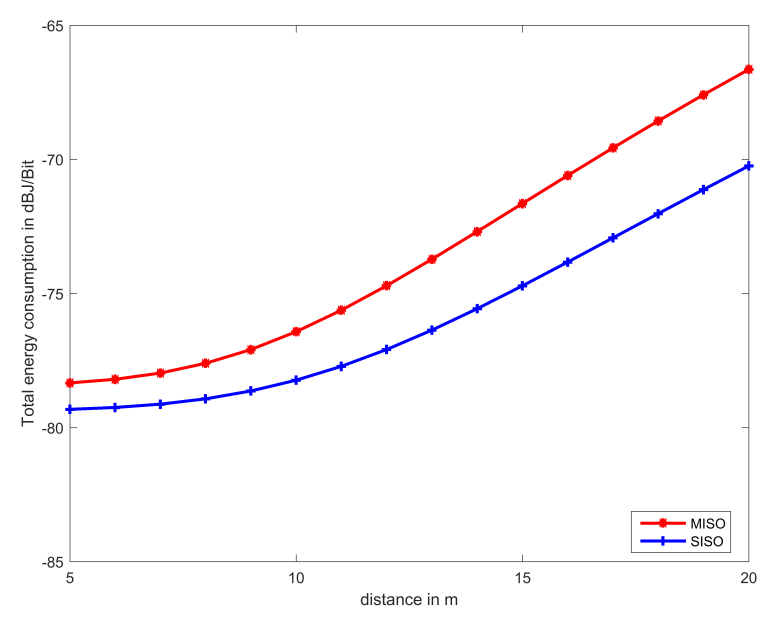

Fig. 5. Total energy consumption for MISO $2 \times 1$ and SISO with $d=[5,20 m], P_{M}=10^{-3}$ and $M=2$ for the NLOS case

the operating range exceeds several meters. In these conditions the transmission energy dominates the overall consumption. That is why the variation of the total consumption is consequent, in the order of $20 \mathrm{~dB}$, in regard to the LOS case where the total energy is constant over the whole range of distances. At the maximum range considered, the MISO system tends to consume more than the corresponding SISO system.

In Fig. 6 and Fig. 7, we depict the total energy consumption of the MISO and the SISO system versus the number of symbols. We use $M \in\{2,4,8,16,32,64\}, P_{M}=10^{3}$, $d=10 \mathrm{~m}, L_{R}=4$ for LOS and NLOS cases respectively.

In both cases LOS and NLOS propagation conditions, the SISO system requests less energy to operate as compared to the MISO system. In the LOS case, the total energy consumption is dominated by the energy linked to the circuitries power. To the contrary, in NLOS conditions, the total energy consumption is dominated by the energy linked to the transmission power.

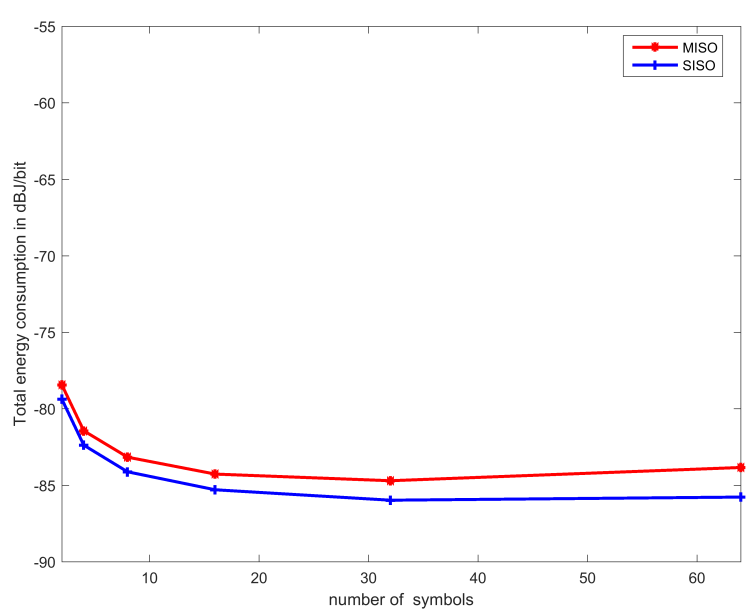

Fig. 6. Total energy consumption for MISO $2 \times 1$ and SISO with $M \in\{2,4,8,16,32,64\}, P_{M}=10^{-3}$ and $d=10 m$ for the LOS case

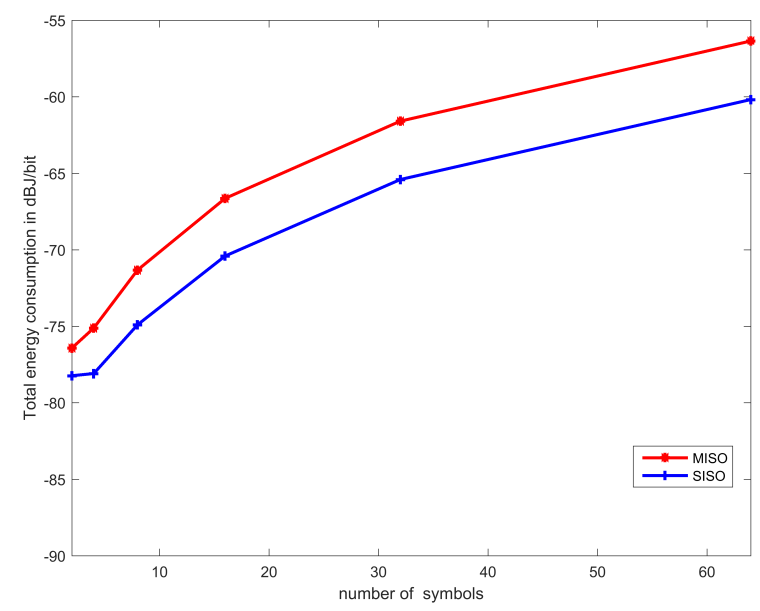

Fig. 7. Total energy consumption for MISO $2 \times 1$ and SISO with $M \in\{2,4,8,16,32,64\}, P_{M}=10^{-3}$ and $d=10 m$ for the NLOS case

According to the operating conditions selected, the model exhibits very different behaviors where energy consumed by circuitries or by the requested transmitted output power can dominate. Other MISO configurations including 4x1 and $6 \times 1$ were also evaluated showing similar general conclusions. Therefore, selecting the defined parameters contained in tables I to III, the model can help evaluate the overall power consumption of the UWB-IR radio link. Moreover, considering the requested supplementary energy consumption, a radiofrequency designer can decide if it is worthwhile to benefitting from the MISO improvements, or using a SISO system.

\section{MISO-UWB VERSUS TR-MISO-UWB}

In this new section, we compare the total energy consumption of the TR-MISO systems and one of the corresponding MISO systems. We operate in an indoor environment and successively in LOS and NLOS propagation conditions. For 
these simulations, we use the same operating parameters than those indicated in previous tables I to III.

In Fig. 8 and Fig. 9, we depict the total energy consumption for the TR-MISO and the MISO systems against the distance for $d=[5,20 m]$. We use $P_{M}=10^{-3}, N_{T}=\{2,4,6\}, L_{T R}=$ 4 and $L_{R}=4$.

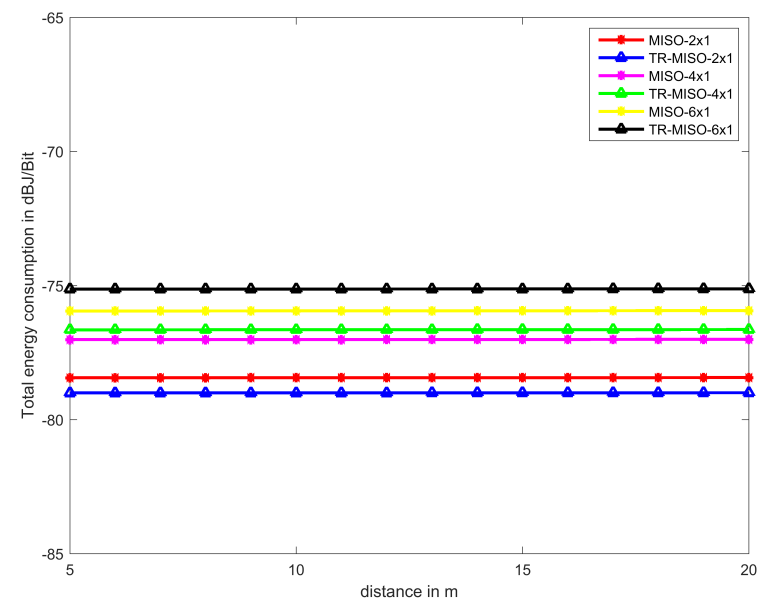

Fig. 8. Total energy consumption for MISO 2x1, MISO 4x1 MISO 6x1 with and without TR where $d=[5,20 m], P_{M}=10^{-3}, M=2$ for the LOS case

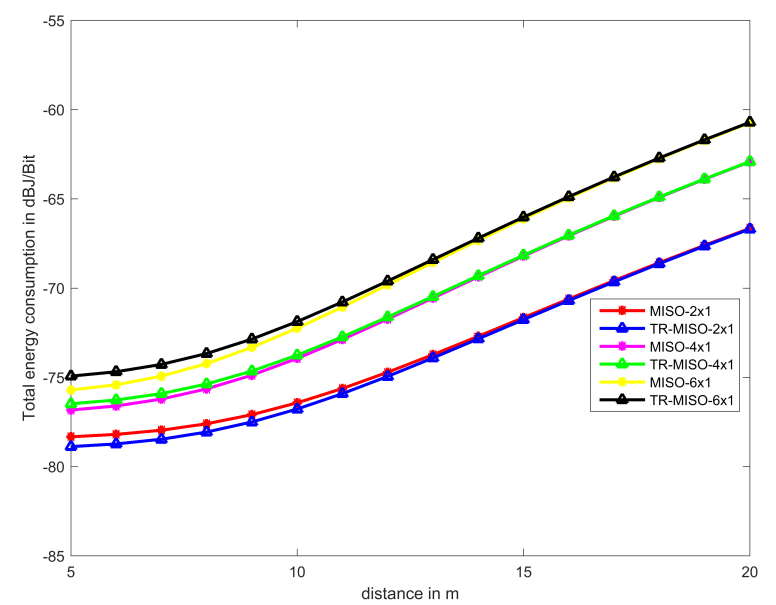

Fig. 9. Total energy consumption for MISO 2x1, MISO 4x1 MISO 6x1 with and without TR where $d=[5,20 m], P_{M}=10^{-3}, M=2$ for the NLOS case

In the LOS case, from the studied electrical energy consumption point of view, we obtain that a two-antenna TRMISO system outperforms the corresponding MISO system. However, this conclusion does not remain valid using 4 or 6 antennas. Indeed, starting from 4 antennas and onward, the advantage given by the TR technique is lost and the TR-MISO UWB system consumes more than the corresponding MISO system. This change is due to the complexity and the need of using more pre-filters and power amplifiers to generate the TR signal. When the number of these different components increases, the energy consumption increases consequently. Therefore, in our selected conditions, using TR with more than two antennas is counterproductive. Moreover, in these LOS conditions, since the requested output power is limited, the consumed energy does not vary significantly as a function of the range and we obtain that the circuitries impose the overall electrical energy consumption.

In the NLOS case, using 2 antennas at the transmitter, we notice a similar, although less contrasted behavior. The same explanation as in the LOS case can be provided. However, since the requested transmitted power is higher in these NLOS propagation conditions, the benefit noticed previously is reduced. Furthermore, the TR-MISO advantage almost disappears at longer ranges, over $d=14 \mathrm{~m}$, where both systems exhibit similar consumptions. As previously, using 4 or 6 antennas, the MISO system consumes less energy at the beginning of the considered communication range and almost the same starting from $d=14 \mathrm{~m}$.

These experiments show that the number of antennas may change the energy efficiency of the system.

In Fig. 10 and Fig. 11, we represent the total energy consumption for the TR-MISO and the MISO systems and we consider different numbers of symbols $M \in$ $\{2,4,8,16,32,64\}$. We use $P_{M}=10^{-3}, d=10 m, N_{T}=$ $\{2,4,6\}, L_{T R}=4$ and $L_{R}=4$ for LOS and NLOS cases respectively.

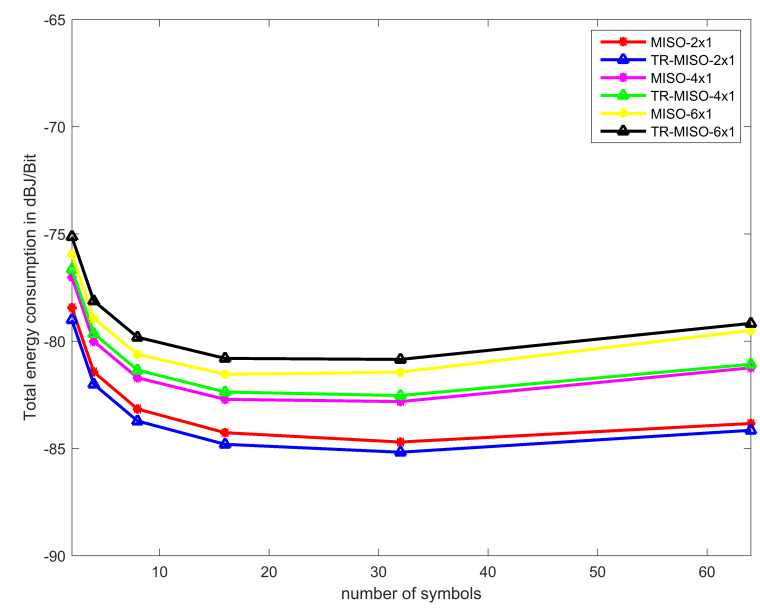

Fig. 10. Total energy consumption for MISO 2x1, MISO 4x1 and MISO $6 \times 1$ with and without TR where $M \in\{2,4,8,16,32,64\}, P_{M}=10^{-3}$ and $d=10 m$ for the LOS case

In the LOS case, using 2 antennas at the transmitter, the TR-MISO consumes less energy than the MISO system for all the considered number of symbols. If we use 4 or 6 antennas, then the MISO system becomes more energy efficient than the TR-MISO system for all the number of symbols considered.

In the NLOS case both systems consume almost the same amount of energy, depending on the value of M. In these conditions the transmission energy dominates the total energy consumed by the system and the circuitry energy does not contribute significantly. 


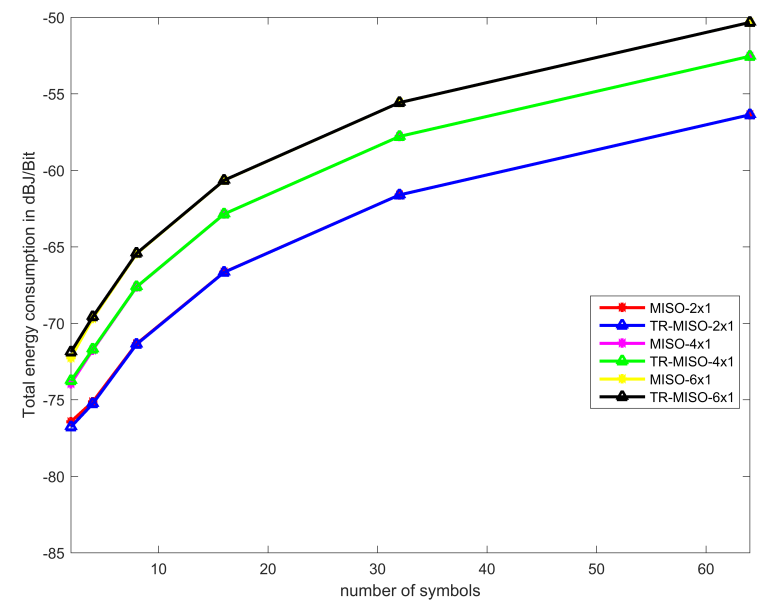

Fig. 11. Total energy consumption for MISO $2 \times 1$, MISO $4 \times 1$ and MISO $6 \times 1$ with and without TR where $M \in\{2,4,8,16,32,64\}, P_{M}=10^{-3}$ and $d=10 m$ for the NLOS case

All these simulations show that the choice of the most efficient system depends on several parameters (data rate, communication technique, and number of antennas). Thus, in our selected operating conditions, the TR-MISO-UWB appears as a better choice if we use 2 antennas while the MISO-UWB becomes more efficient when 4 antennas or more are used. Therefore, if the focusing capability is not important for the developed application, it appears better to use a conventional MISO-UWB system.

\section{CONCLUSION}

We developed in this paper an energetic model that estimates the total energy consumption of a MISO-UWB system in a Nakagami-m channel. We compared the total energy of the MISO system and the SISO system. The study was conducted in an indoor environment, taking into account the propagation specificities presented by the 802.15.4a task group. As expected, the SISO system consumes less than the MISO system, because the latter uses an array of antennas which raises the consumption of the circuitries. We also compared the consumption of TR-MISO and MISO systems for $2 \times 1$, $4 \times 1$ and $6 \times 1$ arrays in the same environment. Using our selected operating conditions, in the case where 2 antennas for transmission are selected, the TR-MISO system outperforms the MISO system in both LOS and NLOS cases. However for $4 \times 1$ and $6 \times 1$ arrays, the MISO system consumes less than the corresponding TR-MISO system. The results showed here can be exploited to design an adaptive energy-aware system alternating between MISO and TR-MISO to achieve the lowest energy consumption possible compatible with the necessary UWB-IR radio link. .

\section{ACKNOWLEDGMENT}

The authors would like to thank Railenium test and research center for supporting this work.

\section{REFERENCES}

[1] T. Kaiser, F. Zheng and E. Dimitrov, An overview of ultra-wide-band systems with MIMO, Proc. IEEE. Vol. 97, no. 2, pp. 285-312, 2009.

[2] A. S. Gowda and J. G. Goldsmith, Energy Efficiency of MIMO Systems, Project Report, Stanford University, Stanford.

[3] A. F. Molisch, MIMO-UWB propagation channel, in Antennas and Propagation (EuCAP), Proc. of the Fourth European Conference on, 2010, pp. 1-6.

[4] G. Tsao, P. Iyamu, L. Petropoulakis, R. Atkinson, I. Andonovic, and I. A. Glover, Measurements of MIMO-UWB indoor channell, in Int. Symp. Signals, Syst. Electron., pp. 1-6, Oct. 2012.

[5] W. Q. Malik and D. J. Edwards, Measured MIMO Capacity and Diversity Gain With Spatial and Polar Arrays in Ultra wideband Channels, IEEE Trans. Commun., vol. 55, no. 12, pp. 2361-2370, Dec. 2007.

[6] H. Nguyen, V. D. Nguyen, T. K. Nguyen, K. Maichalernnukul, F. Zheng, and T. Kaiser, On the Performance of the Time Reversal SMMIMO-UWB System on Correlated Channels, Int. Journal of Antennas and Propagation, doi:10.1155/2012/929018, vol. 2012.

[7] C. Zhou , N. Guo and R. C. Qiu, Time-reversed ultra-wideband (UWB) multiple input multiple output (MIMO) based on measured spatial channels, IEEE Trans. Veh. Technol., vol. 58, no. 6, pp. 2884-2898, 2009.

[8] A. Elabboubi, F. Elbahhar, M. Heddebaut, Y. Elhillali. R. Elassali, Energy efficiency analysis of a TR-UWB system, American Journal of Applied Science, Journal of Computer Science, vol. 12, Issue 1, pp. 19-30, doi: 10.3844 jessp.2016.19.30.

[9] A. F. Molisch, K. Balakrishnan, D. Cassioli, C. Chong, S. Emami, A. Fort, J. Karedal, J. Kunisch, H. Schantz, U. Schuster, K. Siwiak, A comprehensive model for ultrawideband propagation channels of UWB system proposals standard for these applications, in Proc. IEEE Globecom, 2005, in press.

[10] B. Fall, F. Elbahhar, M. Heddebaut, and A. Rivenq, Train accurate localization using ultra wide band radio and time reversal, Int. Journal of Engineering and Innovative Technology, vol. 3, Issue 12, pp. 234-24, 2014.

[11] S. Cui, A. J. Goldsmith, and A. Bahai, Energy-constrained modulation optimization, IEEE Trans. on Wireless Commun., vol. 4, no. 5, pp. 23492360, 2005.

[12] K. Witrisal and M. Pausini, Statistical analysis of UWB channel correlation functions, IEEE Trans. Veh. Technol., vol. 57, Issue 3, 2008.

[13] V. A. Aalo, Performance of maximal-ratio diversity systems in a correlated Nakagami-fading environment, IEEE IEEE Trans. Commun., vol. 3, pp. 2360-2369, 1995.

[14] H. T. Nguyen, Optimal One Bit Time Reversal for UWB Impulse Radio In Multi-User Wireless Communication, in World Academy of Science, Engineering and Technology proceedings, no. 36, pp. 298-304, 2008.

[15] J. G. Proakis, Digital Communications, USA: McGraw-Hill, ch. 5. pp. 257.

[16] W. Q. Malik, Spatial correlation in Ultrawideband channels, IEEE Trans. Wireless Commun., vol. 7, pp. $604-610,2008$

[17] A. Elabboubi, F. Elbahhar, M. Heddebaut, Y. Elhillali, An energy efficiency comparative for UWB modulations over m-Nakagami fading channels with path-loss, in IEEE Int. Conference on Ultra-Wideband, Paris, Sept. 1-3, 2014 